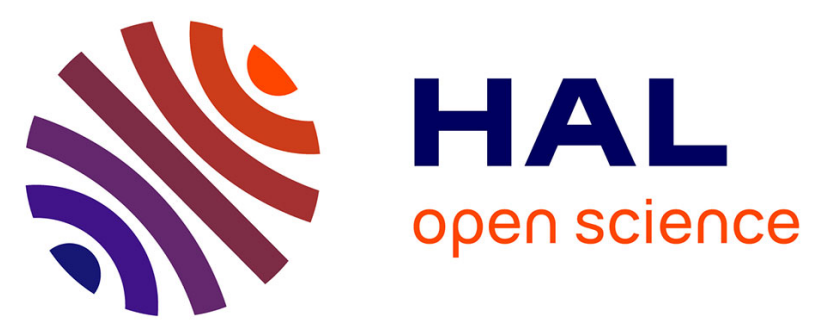

\title{
Sensitivity of Functionals in Problems of Variational Assimilation of Observational Data
}

Victor P. Shutyaev, Francois-Xavier Le Dimet, Valery I. Agoshkov, Evgeny I. Parmuzin

\section{- To cite this version:}

Victor P. Shutyaev, Francois-Xavier Le Dimet, Valery I. Agoshkov, Evgeny I. Parmuzin. Sensitivity of Functionals in Problems of Variational Assimilation of Observational Data. Izvestiya Atmospheric and Oceanic Physics / Izvestiya Rossiiskoi Akademii Nauk Fizika Atmosfery i Okeana, 2015, 51 (3), pp.349-357. 10.1134/S0001433815030111 . hal-01252779

\section{HAL Id: hal-01252779 \\ https://inria.hal.science/hal-01252779}

Submitted on 8 Jan 2016

HAL is a multi-disciplinary open access archive for the deposit and dissemination of scientific research documents, whether they are published or not. The documents may come from teaching and research institutions in France or abroad, or from public or private research centers.
L'archive ouverte pluridisciplinaire HAL, est destinée au dépôt et à la diffusion de documents scientifiques de niveau recherche, publiés ou non, émanant des établissements d'enseignement et de recherche français ou étrangers, des laboratoires publics ou privés. 


\title{
Sensitivity of Functionals in Problems of Variational Assimilation of Observational Data
}

\author{
V. P. Shutyaev ${ }^{a}$, F.-X. Le Dimet ${ }^{b}$, V. I. Agoshkov ${ }^{a}$, and E. I. Parmuzin ${ }^{a}$ \\ ${ }^{a}$ Institute of Computational Mathematics, Russian Academy of Sciences, Moscow, Russia \\ ${ }^{b}$ University of Grenoble, Grenoble, France \\ email: shutyaev@inm.ras.ru,ledimet@imag.fr \\ Received October 8, 2014; in final form, October 17, 2014
}

\begin{abstract}
The problem of the variational assimilation of observational data is stated for a nonlinear evolution model as a problem of optimal control in order to find the function of initial condition. The operator of the model, and consequently the optimal solution, depend on parameters that may contain uncertainties. A functional of the solution of the problem of variational data assimilation is considered. Using the method of second-order adjoint equations, the sensitivity of the functional in respect to the model parameters is studied. The gradient of the functional is expressed through solving a "nonstandard" (nonclassical) problem that involves the coupled system of direct and adjoint equations. The solvability of the nonstandard problem using the Hessian initial functional of observations is studied. Numerical algorithms for solving the problem and computing the gradient of the functional under consideration are developed with respect to the parameters. The results of the studies are applied in the problem of variational data assimilation for a $3 \mathrm{D}$ ocean thermodynamic model.
\end{abstract}

Keywords: variational assimilation, optimal solution, sensitivity of functional

DOI: $10.1134 /$ S0001433815030111

\section{INTRODUCTION}

In mathematically modeling climate changes for different regions of the planet, an important role is played by the theory of sensitivity of selected functionals with respect to the characteristics of the continents, the World Ocean, the initial data, external sources, and the internal parameters of the problem. The problem of climate sensitivity allows us to estimate the quality of models retrospectively and obtain new mechanisms that are responsible for the climate formation based on the actual data.

In the 1970s G.I. Marchuk formulated a fundamental approach to solving the problem of a long-term 1 weather forecast based on so-called adjoint equations for the nonlinear models of the atmosphere and ocean thermohydrodynamics, which make it possible to plot a function of sensitivity for nonstationary nonlinear problems. This approach was the main one to identify the energy active zones of the World Ocean that were studied using the "Sections" program developed and organized by G.I. Marchuk.

1 Later on he developed the theory of adjoint equations and algorithms of disturbances to study the sensitivity of functionals from different classes in the problems of mathematical physics. This was also fruitful for many other scientific areas. This resulted in the appearance of more or less general approaches to studying complex systems and mathematical models.
These approaches constituted the core of the longterm studies by G.I. Marchuk and his scientific school in different branches of mathematics and its applications to diffusion problems, models of environment protection, climate theory and climate changes, mathematical problems of satellite data processing, etc. [1].

In connection with the studies of global changes on planet Earth, it is currently very important to obtain and make efficient use of the measurement data for retrospective analysis in the different areas of knowledge [1-6]. The mathematical model of this problem can be formulated as a problem of assimilation and processing of multidimensional data that include the dependence on the time and spatial variables. This is one of the problems of optimal control. Starting from the works by L.S. Pontryagin, N.N. Krasovskii, J.L. Lions, and G.I. Marchuk, many researchers who are involved in the applications of the optimal control methods for practical solving various problems focus their attention on the statements and studies of the problems of variational data assimilation based on the theory of adjoint equations [3-19]. Along with the 1 development and substantiation of the algorithms of numerically solving the variational assimilation of the observational data, a key role belongs to the properties of the most optimal solution [20-23]. It is extremely 
crucial to consider the problem of sensitivity of optimal solutions to the problems of variational assimilation to the errors of observational data and the parameters of models, which until recently has not been studied very much.

This work considers the problem of studying the sensitivity of functionals of the optimal solution to the problems of the variational assimilation of data to the parameters of the models. Here we propose algorithms for calculating the gradient of functionals based on the 1 method of the second-order adjoint equations. The procedure developed for studying sensitivity is used to study functionals of the optimal solution to the problem of the variational assimilation of observational data on the temperature of an ocean surface for the 3D thermodynamic model.

\section{GENERAL STATEMENT OF THE PROBLEM}

We consider the mathematical model of a physical process described using the evolution problem represented as

$$
\left\{\begin{array}{l}
\frac{\partial \varphi}{\partial t}=F(\varphi, \lambda), t \in(0, T) \\
\left.\varphi\right|_{t=0}=u,
\end{array}\right.
$$

where $\varphi=\varphi(t)$ is the unknown function that belongs to the Hilbert space $X, u \in X$ for any $t, F$ is the nonlinear operator acting from $Y \times Y_{p}$ in $Y=L_{2}(0, T ; X)$, \|\|$_{Y}=(\cdot, \cdot)_{Y}^{1 / 2}$, and $Y_{p}$ is the Hilbert space (the space of model parameters). Let $\varphi \in Y$ be a unique solution to problem (1.1) for the assigned $u \in X$ and $\lambda \in Y_{p}$.

We introduce the functional of observations in the form

$$
\begin{gathered}
J(u)=\frac{1}{2}\left(V_{1}\left(u-u_{0}\right), u-u_{0}\right)_{X} \\
+\frac{1}{2}\left(V_{2}\left(C \varphi-\varphi_{\mathrm{obs}}\right), C \varphi-\varphi_{\mathrm{obs}}\right)_{Y_{\mathrm{obs}}},
\end{gathered}
$$

where $u_{0} \in X$ is the initial approximation of the function of initial state (the background field), $\varphi_{\mathrm{obs}} \in Y_{\mathrm{obs}}$ is the assigned function (the observational data), $Y_{\mathrm{obs}}$ is the Hilbert space (the space of observations), $C: Y \rightarrow Y_{\text {obs }}$ is the linear bounded operator, and $V_{1}: X \rightarrow X$ and $V_{2}: Y_{\text {obs }} \rightarrow Y_{\text {obs }}$ are the symmetrically positive definite operators. In physical problems, the functional (1.2) is nothing other than the mean square deviation from the values observed.

We consider the next problem of variational data assimilation in order to obtain the initial condition (the problem of initialization): to find such $u \in X$ and $\varphi \in Y$ so that they satisfy (1.1) for the assigned $\lambda \in Y_{p}$ and the functional $J(u)$ takes on the least value, i.e.,

$$
\left\{\begin{array}{l}
\frac{\partial \varphi}{\partial t}=F(\varphi, \lambda), t \in(0, T) \\
\left.\varphi\right|_{t=0}=u, \\
J(u)=\inf _{v} J(v) .
\end{array}\right.
$$

The required condition of optimality reduces the problem (1.3) to a so-called system of optimality [14]:

$$
\left\{\begin{array}{l}
\frac{\partial \varphi}{\partial t}=F(\varphi, \lambda), t \in(0, T) \\
\left.\varphi\right|_{t=0}=u,
\end{array}\right.
$$

$$
\left\{\begin{array}{l}
-\frac{\partial \varphi^{*}}{\partial t}-\left(F_{\varphi}^{\prime}(\varphi, \lambda)\right)^{*} \varphi^{*}=-C^{*} V_{2}\left(C \varphi-\varphi_{\text {obs }}\right), t \in(0, T) \\
\left.\varphi^{*}\right|_{t=T}=0
\end{array}\right.
$$

with the unknown functions $\varphi, \varphi^{*}, u$, where $\left(F_{\varphi}^{\prime}(\varphi, \lambda)\right)^{*}$ is the adjoint operator with respect to the derivative of 1 the operator $F$ for $\varphi$ and $C^{*}$ is the adjoint operator with 1 respect to $C$ determined by the equality $(C \varphi, \psi)_{Y_{\mathrm{obs}}}$ $=\left(\varphi, C^{*} \psi\right)_{Y}, \varphi \in Y, \psi \in Y_{\text {obs }}$.

We assume that the system (1.4)-(1.6) has a unique solution. The system (1.4)-(1.6) can be considered a generalized operator equation $\mathscr{F}(U, \lambda)=0$ with the variable $U=\left(\varphi, \varphi^{*}, u\right)$ that contains the entire information. Each component $U$ depends on the parameters $\lambda \in Y_{p}$. The purpose of our work is to study the sensitivity of the functionals of the solution to this generalized equation (the systems (1.4)-(1.6)) with respect to the model parameters.

\section{SENSITIVITY OF FUNCTIONALS}

The mathematical models of Earth sciences often contain parameters that cannot be calculated accurately, since they are the elements of the models of subgrid physical processes, because any model of reality is inaccurate. Therefore, it is important to evaluate the influence of uncertainties in the parameters on the functions obtained after the data are accumulated.

We consider function $G(\varphi, u, \lambda)$, that is assumed to be real-valued and can be considered a functional at $Y \times X \times Y_{p}$. The examples of these functionals will be presented below in section 5 . We will be interested in sensitivity $G$ with respect to $\lambda$ when $\varphi$ and $u$ are obtained after the assimilation of the data from the system of optimality (1.4) - (1.6). As is known [1, 24, 
25], sensitivity is determined by gradient $G$ with respect to $\lambda$, which is a functional derivative:

$$
\frac{d G}{d \lambda}=\frac{\partial G}{\partial \varphi} \frac{\partial \varphi}{\partial \lambda}+\frac{\partial G}{\partial u} \frac{\partial u}{\partial \lambda}+\frac{\partial G}{\partial \lambda} .
$$

If $\delta \lambda$ is the variation of $\lambda$, from the optimality system we obtain

$$
\begin{gathered}
\left\{\begin{array}{l}
\frac{\partial \delta \varphi}{\partial t}=F_{\varphi}^{\prime}(\varphi, \lambda) \delta \varphi+F_{\lambda}^{\prime}(\varphi, \lambda) \delta \lambda, t \in(0, T) \\
\left.\delta \varphi\right|_{t=0}=\delta u,
\end{array}\right. \\
\left\{\begin{array}{l}
-\frac{\partial \delta \varphi^{*}}{\partial t}-\left(F_{\varphi}^{\prime}(\varphi, \lambda)\right)^{*} \delta \varphi^{*} \\
-\left(F_{\varphi \varphi}^{\prime \prime}(\varphi, \lambda) \delta \varphi+F_{\varphi \lambda}^{\prime \prime}(\varphi, \lambda) \delta \lambda\right)^{*} \varphi^{*}=-C^{*} V_{2} C \delta \varphi, \\
\left.\delta \varphi^{*}\right|_{t=T}=0, \\
V_{1} \delta u-\left.\delta \varphi^{*}\right|_{t=0}=0
\end{array}\right.
\end{gathered}
$$

and

$$
\begin{gathered}
\left(\frac{d G}{d \lambda}, \delta \lambda\right)_{Y_{p}} \\
=\left(\frac{\partial G}{\partial \varphi}, \delta \varphi\right)_{Y}+\left(\frac{\partial G}{\partial u}, \delta u\right)_{X}+\left(\frac{\partial G}{\partial \lambda}, \delta \lambda\right)_{Y_{p}},
\end{gathered}
$$

where $\delta \varphi, \delta \varphi^{*}$, and $\delta u$ are the derivatives of $\varphi, \varphi^{*}$, and $u$ in the direction of $\delta \lambda$ (for example, $\delta \varphi=\frac{\partial \varphi}{\partial \lambda} \delta \lambda$ ).

To calculate the gradient $\frac{d G}{d \lambda}$, we introduce three 1 adjoint variables $P_{1} \in Y, P_{2} \in Y$, and $P_{3} \in X$. By scalar multiplying equation (2.2) by $P_{1}$, equation (2.3) by $P_{2}$, and equation (2.4) by $P_{3}$ and adding them, we obtain

$$
\begin{gathered}
\left(\delta \varphi,-\frac{\partial P_{1}}{\partial t}-\left(F_{\varphi}^{\prime}(\varphi, \lambda)\right)^{*} P_{1}-\left(F_{\varphi \varphi}^{\prime \prime}(\varphi, \lambda) P_{2}\right)^{*} \varphi^{*}\right. \\
\left.+C^{*} V_{2} C P_{2}\right)_{Y}+\left(\left.\delta \varphi\right|_{t=T},\left.P_{1}\right|_{t=T}\right)_{X}+\left(\delta \varphi^{*}, \frac{\partial P_{2}}{\partial t}\right. \\
\left.-F_{\varphi}^{\prime}(\varphi, \lambda) P_{2}\right)_{Y}+\left(\left.\delta \varphi^{*}\right|_{t=0},\left.P_{2}\right|_{t=0}-P_{3}\right)_{X} \\
+\left(\delta u,-\left.P_{1}\right|_{t=0}+V_{1} P_{3}\right)_{X}+\left(\delta \lambda,-\left(F_{\lambda}^{\prime}(\varphi, \lambda)\right)^{*} P_{1}\right. \\
\left.-\left(F_{\varphi \lambda}^{\prime \prime}(\varphi, \lambda) P_{2}\right)^{*} \varphi^{*}\right)_{Y_{p}}=0 .
\end{gathered}
$$

Assume that

$$
\begin{gathered}
-\frac{\partial P_{1}}{\partial t}-\left(F_{\phi}^{\prime}(\phi, \lambda)\right)^{*} P_{1} \\
-\left(F_{\phi \phi}^{\prime \prime}(\phi, \lambda) P_{2}\right)^{*} \phi^{*}+C^{*} V_{2} C P_{2}=\frac{\partial G}{\partial \phi}
\end{gathered}
$$

and

$$
\begin{gathered}
-\left.P_{1}\right|_{t=0}+V_{1} P_{3}=\frac{\partial G}{\partial u},\left.\quad P_{1}\right|_{t=T}=0, \\
\frac{\partial P_{2}}{\partial t}-F_{\varphi}^{\prime}(\varphi, \lambda) P_{2}=0,\left.\quad P_{2}\right|_{t=0}-P_{3}=0 .
\end{gathered}
$$

We can eliminate the variable $P_{3}$ by $P_{3}=\left.P_{2}\right|_{t=0}$ and obtain the initial condition for $P_{2}$ in the form $\left.V_{1} P_{2}\right|_{t=0}=$ $\frac{\partial G}{\partial u}+\left.P_{1}\right|_{t=0}$. Therefore, if $P_{1}$ and $P_{2}$ satisfy the system of equations

$$
\left\{\begin{array}{l}
-\frac{\partial P_{1}}{\partial t}-\left(F_{\varphi}^{\prime}(\varphi, \lambda)\right)^{*} P_{1} \\
-\left(F_{\varphi \varphi}^{\prime \prime}(\varphi, \lambda) P_{2}\right)^{*} \varphi^{*}+C^{*} V_{2} C P_{2}=\frac{\partial G}{\partial \varphi}, t \in(0, T) \\
\left.P_{1}\right|_{t=T}=0 \\
\left\{\begin{array}{l}
\frac{\partial P_{2}}{\partial t}-F_{\varphi}^{\prime}(\varphi, \lambda) P_{2}=0, t \in(0, T) \\
\left.V_{1} P_{2}\right|_{t=0}=\frac{\partial G}{\partial u}+\left.P_{1}\right|_{t=0}
\end{array}\right.
\end{array}\right.
$$

from (2.6) we obtain

$$
\begin{gathered}
\left(\frac{\partial G}{\partial \varphi}, \delta \varphi\right)_{Y}+\left(\frac{\partial G}{\partial u}, \delta u\right)_{X} \\
=\left(\delta \lambda,\left(F_{\lambda}^{\prime}(\varphi, \lambda)\right)^{*} P_{1}+\left(F_{\varphi \lambda}^{\prime \prime}(\varphi, \lambda) P_{2}\right)^{*} \varphi^{*}\right)_{Y_{p}},
\end{gathered}
$$

and calculate the gradient of the functional $G$ by the formula

$$
\frac{d G}{d \lambda}=\left(F_{\lambda}^{\prime}(\varphi, \lambda)\right)^{*} P_{1}+\left(F_{\varphi \lambda}^{\prime \prime}(\varphi, \lambda) P_{2}\right)^{*} \varphi^{*}+\frac{\partial G}{\partial \lambda} .
$$

We obtained the coupled system for the first-order differential equations (2.7) and (2.8) with respect to time. The first problem is considered under the final condition (the adjoint problem) and the second problem under the initial condition (the direct problem), which depends on the initial value of the first equation solution: this is a nonstandard (nonclassical) system.

\section{ALGORITHM FOR SOLVING A NONSTANDARD PROBLEM}

We consider a nonstandard problem (2.7) $-(2.8)$ and write it in the equivalent form:

$$
\left\{\begin{array}{l}
-\frac{\partial P_{1}}{\partial t}-\left(F_{\varphi}^{\prime}(\varphi, \lambda)\right)^{*} P_{1}-\left(F_{\varphi \varphi}^{\prime \prime}(\varphi, \lambda) P_{2}\right)^{*} \varphi^{*} \\
+C^{*} V_{2} C P_{2}=\frac{\partial G}{\partial \varphi}, t \in(0, T) \\
\left.P_{1}\right|_{t=T}=0
\end{array}\right.
$$

$$
\begin{aligned}
& \left\{\begin{array}{l}
\frac{\partial P_{2}}{\partial t}-F_{\varphi}^{\prime}(\varphi, \lambda) P_{2}=0, t \in(0, T) \\
\left.P_{2}\right|_{t=0}=v,
\end{array}\right. \\
& V_{1} V-\left.P_{1}\right|_{t=0}=\frac{\partial G}{\partial u} .
\end{aligned}
$$

IZVESTIYA, ATMOSPHERIC AND OCEANIC PHYSICS Vol. $51 \quad$ No. 32015 
Here we have three unknown values: $v \in X, P_{1}$, and $P_{2} \in Y$. We rewrite the system (3.1)-(3.3) as an operator equation for $V$. First we determine the operator $\mathcal{H}$, acting in the space $X$ by successively solving the following problems:

$$
\begin{gathered}
\left\{\begin{array}{l}
\frac{\partial \phi}{\partial t}-F_{\varphi}^{\prime}(\varphi, \lambda) \phi=0, t \in(0, T) \\
\left.\phi\right|_{t=0}=w,
\end{array}\right. \\
\left\{\begin{array}{l}
-\frac{\partial \phi^{*}}{\partial t}-\left(F_{\varphi}^{\prime}(\varphi, \lambda)\right)^{*} \phi^{*}-\left(F_{\varphi \varphi}^{\prime \prime}(\varphi, \lambda) \phi\right)^{*} \varphi^{*} \\
=-C^{*} V_{2} C \phi, t \in(0, T) \\
\left.\phi^{*}\right|_{t=T}=0,
\end{array}\right. \\
\mathcal{H}_{w}=V_{1} w-\left.\phi^{*}\right|_{t=0} .
\end{gathered}
$$

Then the problem (3.1)-(3.3) is equivalent to the following problem in $X$ :

$$
\mathscr{H}_{V}=\Phi
$$

with the right part

$$
\Phi=\frac{\partial G}{\partial u}+\left.\tilde{\phi}^{*}\right|_{t=0}
$$

1 where $\tilde{\phi}^{*}$ is the solution to the adjoint problem

$$
\left\{\begin{array}{l}
-\frac{\partial \tilde{\phi}^{*}}{\partial t}-\left(F_{\varphi}^{\prime}(\varphi, \lambda)\right)^{*} \tilde{\phi}^{*}=\frac{\partial G}{\partial \varphi}, t \in(0, T) \\
\left.\tilde{\phi}^{*}\right|_{t=T}=0 .
\end{array}\right.
$$

It is easy to realize that the operator $\mathscr{H}$, calculated from (3.4)-(3.6) is a Hessian of the initial functional $J$ considered for the optimal solution $u$ of the problem (1.4)-(1.6): $J^{\prime \prime}(u)=\mathscr{H}$. On the assumption that $\mathcal{H}$ is positively determined, Eq. (3.7) is correct and is solvable in $X$ everywhere; i.e., any $\Phi \in X$ has a unique solution $v \in X$ and the estimate

is valid.

$$
\|\nabla\|_{X} \leq c\|\Phi\|_{X}, \quad c=\text { const }>0 .
$$

Therefore, on the assumption that $J^{\prime \prime}(u)$ is calculated positively for the optimal solution (a sufficient condition for the minimum of the functional), nonstandard problem (2.7)-(2.8) has a unique solution of $P_{1}, P_{2} \in Y$.

Based on the above, we present the following algorithm for solving a nonstandard problem.

1 1. We solve the adjoint problem for $\frac{\partial G}{\partial u} \in X, \frac{\partial G}{\partial \varphi} \in Y$

$$
\left\{\begin{array}{l}
-\frac{\partial \tilde{\phi}^{*}}{\partial t}-\left(F_{\varphi}^{\prime}(\varphi, \lambda)\right)^{*} \tilde{\phi}^{*}=\frac{\partial G}{\partial \varphi}, t \in(0, T) \\
\left.\tilde{\phi}^{*}\right|_{t=T}=0
\end{array}\right.
$$

and assume that $\Phi=\frac{\partial G}{\partial u}+\left.\tilde{\phi}^{*}\right|_{t=0}$.
2. We find $v$ as a solution to the equation $\mathscr{H}_{V}=\Phi$ with a Hessian of the initial functional $J$ calculated by (3.4)-(3.6).

3. We successively solve the direct and the adjoint 1 problems

$$
\begin{gathered}
\left\{\begin{array}{l}
\frac{\partial P_{2}}{\partial t}-F_{\varphi}^{\prime}(\varphi, \lambda) P_{2}=0, t \in(0, T) \\
\left.P_{2}\right|_{t=0}=v,
\end{array}\right. \\
\left\{\begin{array}{l}
-\frac{\partial \tilde{P}_{1}}{\partial t}-\left(F_{\varphi}^{\prime}(\varphi, \lambda)\right)^{*} \tilde{P}_{1}-\left(F_{\varphi \varphi}^{\prime \prime}(\varphi, \lambda) P_{2}\right)^{*} \varphi^{*} \\
+C^{*} V_{2} C P_{2}=0, t \in(0, T) \\
\left.\tilde{P}_{1}\right|_{t=T}=0
\end{array}\right.
\end{gathered}
$$

and assume that $P_{1}=\tilde{P}_{1}+\tilde{\phi}^{*}$.

Thus, we obtain $P_{1}, P_{2} \in Y$ as the solutions to problem (2.7)-(2.8).

We use the presented approach in the following sections to consider the application to the problem of the variational data assimilation for the $3 \mathrm{D}$ model of the ocean thermodynamics.

\section{THE PROBLEM OF VARIATIONAL DATA ASSIMILATION FOR THE MODEL OF OCEAN THERMODYNAMICS}

We consider the problem of ocean thermodynamics in the form $[26,27]$ :

$$
\begin{gathered}
T_{t}+(\bar{U}, \mathrm{Grad}) T-\operatorname{Div}\left(\hat{a}_{T} \operatorname{Grad} T\right)=f_{T} \text { in } D \times\left(t_{0}, t_{1}\right), \\
T=T_{0} \text { at } t=t_{0} \text { in } D, \\
-v_{T} \frac{\partial T}{\partial z}=Q \text { on } \Gamma_{S} \times\left(t_{0}, t_{1}\right), \\
\frac{\partial T}{\partial N_{T}}=0 \text { on } \Gamma_{w, c} \times\left(t_{0}, t_{1}\right), \\
\bar{U}_{n}^{-} T+\frac{\partial T}{\partial N_{T}}=\bar{U}_{n}^{-} d_{T}+Q_{T} \text { on } \Gamma_{w, o p} \times\left(t_{0}, t_{1}\right), \\
\frac{\partial T}{\partial N_{T}}=0 \text { on } \Gamma_{H} \times\left(t_{0}, t_{1}\right),
\end{gathered}
$$

where $T=T(x, y, z, t)$ is the unknown function of the temperature; $t \in\left(t_{0}, t_{1}\right),(x, y, z) \in D=\Omega(0, H), \Omega \subset R^{2}$, and $H=H(x, y)$ are the function of the bottom relief; $Q=Q(x, y, t)$ is the heat flux through the upper boundary; and $\bar{U}=(u, v, w), \hat{a}_{T}=\operatorname{diag}\left(\left(a_{T}\right)_{i i}\right),\left(a_{T}\right)_{11}=\left(a_{T}\right)_{22}=$ $\mu_{T}$, and $\left(a_{T}\right)_{33}=v_{T}, f_{T}=f_{T}(x, y, z, t)$ are the assigned functions. The region boundary $\Gamma \equiv \partial D$ is represented as an aggregate of four noncrossing parts $\Gamma_{S}, \Gamma_{w, o p}$, $\Gamma_{w, c}$, and $\Gamma_{H}$, where $\Gamma_{S}=\Omega$ (undisturbed surface of the ocean), $\Gamma_{w, o p}$ is the liquid (open) part of the vertical lateral boundary, $\Gamma_{w, c}$ is the solid part of the vertical lateral boundary, and $\Gamma_{H}$ is the ocean bottom. Here, 
Grad denotes a typical gradient of the field in a 3D space. The other designations and detailed description of the problem statement can be found in $[27,8]$. tion:

Problem (4.1) can be written as an operator equa-

$$
T_{t}+L T=\mathscr{F}_{F}+B Q, \quad t \in\left(t_{0}, t_{1}\right), \quad T=T_{0}, \quad t=t_{0},
$$

where the equality is realized in a weak sense, viz.:

$$
\left(T_{t}, \hat{T}\right)+(L T, \hat{T})=\mathscr{F}(\hat{T})+(B Q, \hat{T}) \forall \hat{T} \in W_{2}^{1}(D),
$$

Here, $L, \mathscr{F}$, and $B$ are determined by the following relationships:

$$
\begin{aligned}
(L T, \hat{T}) & \equiv \int_{a}(-T \operatorname{Div}(\bar{U} \hat{T})) d D+\int_{\Gamma_{w, o p}} \bar{U}_{n}^{(+)} T \hat{T} d \Gamma \\
& +\int_{D} \hat{a}_{T} \operatorname{Grad}(T) \cdot \operatorname{Grad}(\hat{T}) d D, \\
\mathscr{F}(\hat{T}) & =\int_{\Gamma_{w, o p}}\left(Q_{T}+\bar{U}_{n}^{(-)} d_{T}\right) \hat{T} d T+\int_{D} f_{T} \hat{T} d D, \\
\left(T_{t}, \hat{T}\right) & =\int_{D} T_{t} \hat{T} d D,(B Q, \hat{T})=\left.\int_{\Omega} Q \hat{T}\right|_{z=0} d \Omega,
\end{aligned}
$$

and the functions $\hat{a}_{T}, Q_{T}, f_{T}$, and $Q$ are such that equality (4.3) makes sense. The properties of the operator $L$ were studied in [8].

We consider the problem on the assimilation of data on the ocean surface temperature. Assume that the function $T_{0} \in L_{2}(D)$ in problem (4.1) is unknown. We assign the function of observational data $T_{\text {obs }}(x, y, t)$ on $\bar{\Omega} \equiv \Omega \cup \partial \Omega$ at $t \in\left(t_{0}, t_{1}\right)$, that is the approximation to the function of a surface temperature on $\Omega$, i.e., to $\left.T\right|_{z=0}$ in its physical sense. Let $T_{\text {obs }} \in L_{2}\left(\Omega \times\left(t_{0}, t_{1}\right)\right)$; however, the function $T_{\text {obs }}$ cannot be smoother; therefore it cannot be used as a boundary condition on $\Gamma_{S}$. There may present a case when $T_{\text {obs }}$ exists only on a certain subset from $\Omega \times\left(t_{0}, t_{1}\right)$, whose characteristic function is denoted as $m_{0}$.

We consider the problem of the assimilation of the data on the surface temperature in the form: to find such $T$ and $T_{0}$ that

$$
\left\{\begin{array}{l}
T_{t}+L T=F+B Q, \text { в } D\left(t_{0}, t_{1}\right), \\
T=T_{0}, t=t_{0} \\
J\left(T_{0}\right)=\inf _{V} J(v)
\end{array}\right.
$$

where

$$
\begin{gathered}
J\left(T_{0}\right)=\frac{\alpha}{2} \int_{D}\left|T_{0}-T^{(0)}\right|^{2} d D \\
+\frac{1}{2} \int_{t_{0} \Omega}^{t_{1}} \int_{0} m_{0}|T|_{z=0}-\left.T_{\mathrm{obs}}\right|^{2} d \Omega d t,
\end{gathered}
$$

and $T^{(0)}=T^{(0)}(x, y, z)$ is the assigned function, $\alpha$ $=$ const $>0$.

For $\alpha>0$, the stated problem of variational data assimilation has a unique solution. The existence of the optimal solution follows from the classical results of the theory of extremum problems, as it is easy to show that solving the problem (4.1) depends continuously on the initial condition $T_{0}$ (there are a priori estimates in the respective functional spaces). We should mention that, at $\alpha=0$, the problem may not have a unique solution.

According to the required condition $\frac{d J}{d T_{0}}=0$, the system of optimality that determines the solution of the formulated problem of the variational data assimilation has the form

$$
\begin{gathered}
-\left(T^{*}\right)_{t}+L^{*} T^{*}=-B m_{0}\left(T-T_{\mathrm{obs}}\right) \text { in } D \times\left(t_{0}, t_{1}\right), \\
T^{*}=0, \text { for } t=t_{1}, \\
\alpha\left(T_{0}-T^{(0)}\right)-T^{*}=0 \text { for } t=t_{0},
\end{gathered}
$$

where $L^{*}$ is the $L$-adjoint operator.

Later on, we study the problem of sensitivity of the functionals of the optimal solution $T_{0}$ to the model parameters, viz. to the heat fluxes on the surface $Q$.

\section{FUNCTIONALS OF TEMPERATURE AND THEIR SENSITIVITY}

We consider the problem of sensitivity of functionals of the optimal solution $T_{0}$ to the parameters of the system (4.6)-(4.8). We introduce the functional

$$
G(T)=\int_{t_{0}}^{t_{1}} d t \int_{\Omega} F^{*}(x, y, t) T(x, y, 0, t) d \Omega,
$$

where $F^{*}(x, y, t)$ is a certain weight function that is related to the field of temperature on the surface $z=0$. For example, if we want to determine the average temperate in a certain water area of the ocean $\omega$ for $z=0$ in the range $\bar{t}-\tau \leq t \leq \bar{t}$, the function

$$
F^{*}(x, y, t)=\left\{\begin{array}{l}
1 /(\tau m e s \omega) \text { if }(x, y) \in \omega, \\
\bar{t}-\tau \leq t \leq \bar{t} \\
0 \text { in the opposite case }
\end{array}\right.
$$

is selected as $F^{*}$, where mes $\omega$ usually denotes the area of the region $\omega$. Functional (5.1) takes on the form:

$$
G(T)=\frac{1}{\tau} \int_{\bar{t}-\tau}^{\bar{t}} d t\left(\frac{1}{m e s \omega} \int_{\omega} T(x, y, 0, t) d \Omega\right) .
$$

Expression (5.3) is an average temperature in the interval $\bar{t}-\tau \leq t \leq \bar{t}$ for the selected region $\omega$. Functionals of such type are most interesting in the theory of climate changes [1]. 
In our designations, functional (5.1) can be written as

$$
G(T)=\int_{t_{0}}^{t_{1}}\left(B F^{*}, T\right) d t=\left(B F^{*}, T\right)_{Y}, Y=L_{2}\left(D \times\left(t_{0}, t_{1}\right)\right) .
$$

We are interested in the sensitivity of the functional $G(T)$ obtained for $T$ after the assimilation of the observational data with respect to the heat fluxes on the ocean surface $Q$.

The sensitivity is represented by the gradient of the functional $G$ with respect to $Q$ :

$$
\frac{d G}{d Q}=\frac{\partial G}{\partial T} \frac{\partial T}{\partial Q}
$$

Since $G(T)=\left(B F^{*}, T\right)_{Y}$, then $\left(\frac{\partial G}{\partial T}, \delta T\right)_{Y}=\left(B F^{*}, \delta T\right)_{Y}$ and $\frac{\partial G}{\partial T}=B F^{*}$. In accordance with Section 2, we can show that if $P_{1}$ and $P_{2}$ are the solutions to the system of equations

$$
\begin{gathered}
\left\{\begin{array}{l}
-\frac{\partial P_{1}}{\partial t}+L^{*} P_{1}+B m_{0} P_{2}=B F^{*}, t \in(0, T) \\
\left.P_{1}\right|_{t=T}=0,
\end{array}\right. \\
\left\{\begin{array}{l}
\frac{\partial P_{2}}{\partial t}+L P_{2}=0, t \in(0, T) \\
\left.\alpha P_{2}\right|_{t=0}=\left.P_{1}\right|_{t=0},
\end{array}\right.
\end{gathered}
$$

the gradient of the $G$ functional is assigned by the formula

$$
\frac{d G}{d Q}=\left.P_{1}\right|_{z=0} .
$$

As for the general nonlinear case, we again obtain the coupled system of two first-order differential equations with respect to time (5.5) and (5.6). For the first 1 problem there is a final condition (an adjoint problem) and for the second one an initial condition (a direct problem), which depends on the initial value of the solution to the first equation: this is again a nonstandard system, even in this linear case.

We represent the nonstandard problem (5.5)-(5.6) in the equivalent form:

$$
\begin{gathered}
\left\{\begin{array}{c}
-\frac{\partial P_{1}}{\partial t}+L^{*} P_{1}+B m_{0} P_{2}=B F^{*}, t \in(0, T) \\
\left.P_{1}\right|_{t=T}=0,
\end{array}\right. \\
\left\{\begin{array}{l}
\frac{\partial P_{2}}{\partial t}+L P_{2}=0, t \in(0, T) \\
\left.P_{2}\right|_{t=0}=v, \\
\alpha v-\left.P_{1}\right|_{t=0}=0,
\end{array}\right.
\end{gathered}
$$

where we already have three unknown values: $v \in L_{2}(D), P_{1}$, and $P_{2} \in Y$. We proceed from (5.8)-
(5.10) to one operator equation for $v$. For this purpose, we introduce the operator $\mathscr{H}$ acting on the functions $w \in L_{2}(D)$ and calculated by solving the following problems consecutively:

$$
\begin{gathered}
\left\{\begin{array}{l}
\frac{\partial \phi}{\partial t}+L \phi=0, t \in(0, T) \\
\left.\phi\right|_{t=0}=w,
\end{array}\right. \\
\left\{\begin{array}{c}
-\frac{\partial \phi^{*}}{\partial t}+L^{*} \phi^{*}=-B m_{0} \phi, t \in(0, T) \\
\left.\phi^{*}\right|_{t=T}=0, \\
\mathscr{H}_{w}=\alpha w-\left.\phi^{*}\right|_{t=0} .
\end{array}\right.
\end{gathered}
$$

Then the system (5.8)-(5.10) is equivalent to the following operator equation in the space $L_{2}(D)$ :

$$
\mathscr{H}_{V}=\Phi
$$

with the right part

$$
\Phi=\left.\tilde{\phi}^{*}\right|_{t=0},
$$

where $\tilde{\phi}^{*}$ is the solution to the adjoint problem:

$$
\left\{\begin{array}{l}
-\frac{\partial \tilde{\varphi}^{*}}{\partial t}+L^{*} \tilde{\varphi}^{*}=B F^{*}, t \in(0, T) \\
\left.\tilde{\varphi}^{*}\right|_{t=T}=0 .
\end{array}\right.
$$

The operator $\mathscr{H}$, calculated by equalities (5.11)(5.13) is the Hessian of the initial functional $J$ from (4.5). We can show that, if $\alpha>0$, the operator $\mathcal{H}$ is positive and equation (5.14) is correct and is solvable everywhere in $L_{2}(D)$; i.e., for each $\Phi$ there is a unique solution $V \in L_{2}(D)$ and the apriori estimate 2 $\|v\|_{X} \leq c\|\Phi\|_{X}, c=$ const $>0$ is valid. Therefore, problem (5.5)-(5.6) has a unique solution $P_{1}, P_{2} \in Y$.

Based on the above, we formulate the following algorithm for calculating the gradient of the functional $G(T)$.

1. We solve the adjoint problem for $F^{*} \in Y_{p}$

$$
\left\{\begin{array}{l}
-\frac{\partial \tilde{\phi}^{*}}{\partial t}+L^{*} \tilde{\phi}^{*}=B F^{*}, t \in(0, T) \\
\left.\tilde{\phi}^{*}\right|_{t=T}=0
\end{array}\right.
$$

and assume $\Phi=\left.\tilde{\phi}^{*}\right|_{t=0}$.

2. We find $v$ as the solution to the problem $\mathscr{H}_{V}=\Phi$ with the Hessian of the initial functional $J$ calculated by formulas (5.11)-(5.13). 


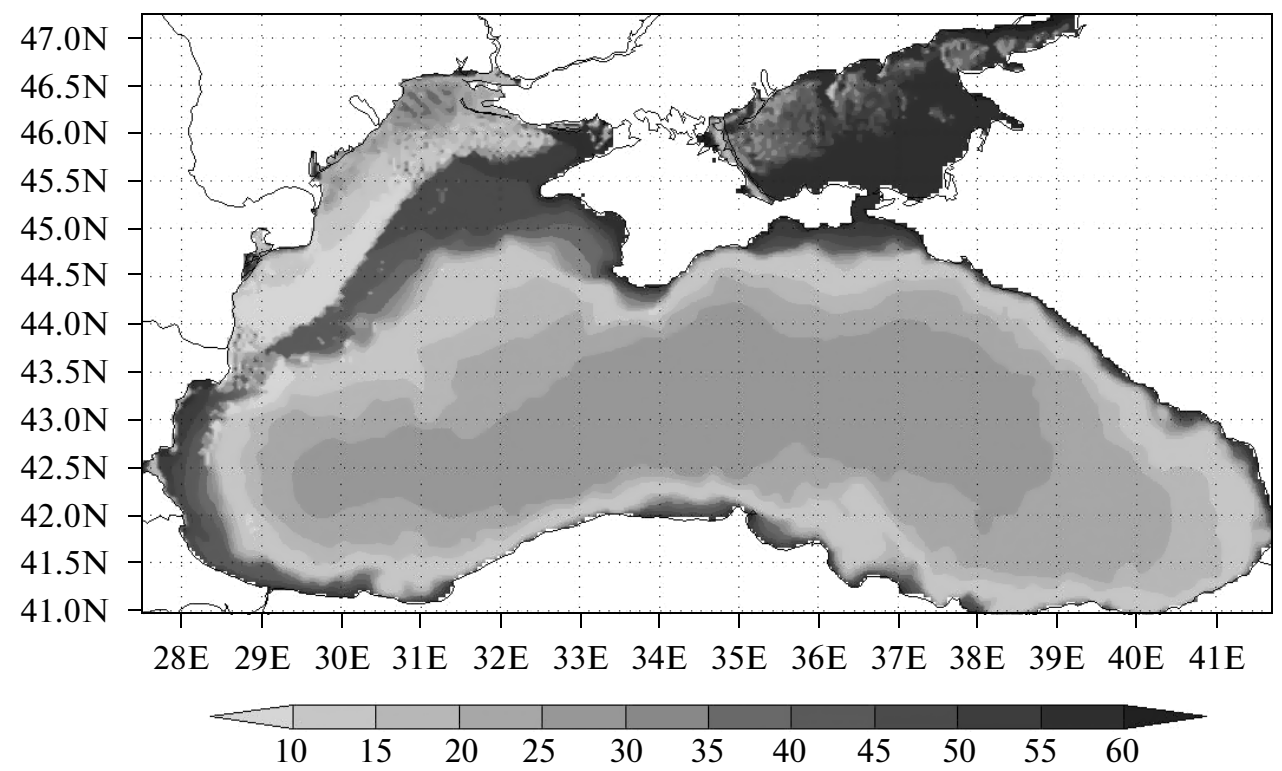

Fig. 1. Hessian eigenvalues after 250 calculation steps.

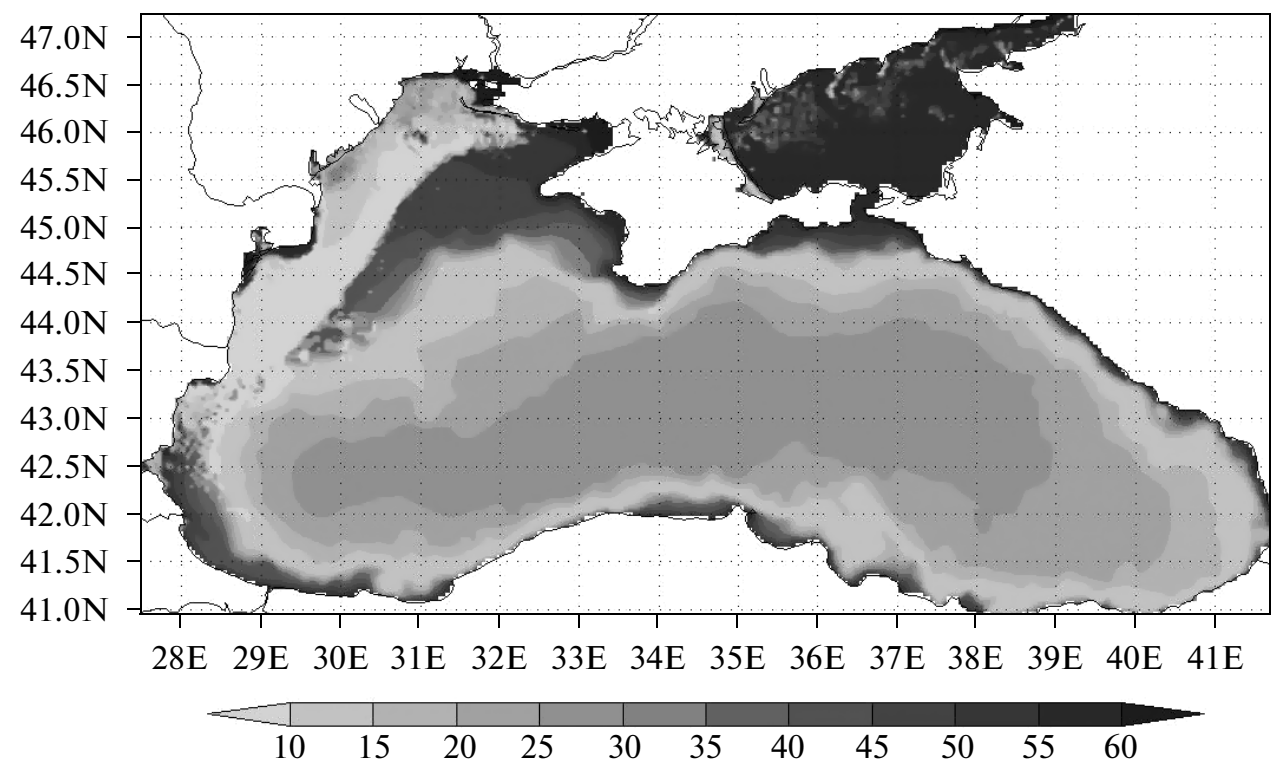

Fig. 2. Hessian eigenvalues after 500 calculation steps.

13 . We solve the direct and the adjoint problems consecutively

$$
\begin{gathered}
\left\{\begin{array}{l}
\frac{\partial P_{2}}{\partial t}+L P_{2}=0, t \in(0, T) \\
\left.P_{2}\right|_{t=0}=v,
\end{array}\right. \\
\left\{\begin{array}{l}
-\frac{\partial \tilde{P}_{1}}{\partial t}+L^{*} \tilde{P}_{1}+B m_{0} P_{2}=0, t \in(0, T) \\
\left.\tilde{P}\right|_{t=T}=0,
\end{array}\right.
\end{gathered}
$$

and assume $P_{1}=\tilde{P}_{1}+\tilde{\phi}^{*}$.

4. We calculate the gradient of the functional by the equation

$$
\frac{d G}{d Q}=\left.P_{1}\right|_{z=0} .
$$

Algorithm (5.17)-(5.20) makes it possible to estimate the sensitivity of the functional related to the temperature after the assimilation of the observational 
data with respect to the heat fluxes on the ocean surface.

In the realization of algorithm $(5.17)-(5.20)$, it is important to solve the equation $\mathscr{H}_{V}=\Phi$ with the Hes$\operatorname{sian} \mathcal{H}$ of the initial functional $J$. If $\alpha$ is small, operator H can be poorly determined, which is caused by the small values of some of its eigenvalues. The result of numerical calculation of the eigenvalues of Hessian $\mathscr{H}$ based on the example of the Black Sea water area is presented in Figs. 1 and 2, from which it follows that the solution to equation $\mathscr{H}_{V}=\Phi$ can be unstable at the points lying near the coastal frontier. The detailed description of the numerical experiment for the problem of the data assimilation in the water area of the Black Sea can be found in [28].

\section{CONCLUSIONS}

The problem of the variational assimilation of the observational data (the problem of initialization of geophysical fields) can be formulated as a problem of optimal control in order to find the function of the initial condition. The optimal solution obtained as a result of assimilation depends on the model parameters and the external sources that may contain uncertainties. This work presents the functionals of the solutions to the problem of variational data assimilation and the study of the sensitivity of the functionals with respect to the parameters. It is shown that the calculation of the gradient of the functionals is reduced to solving a nonstandard (nonclassical) problem that 1 includes direct and adjoint equations of the model with mutual initial conditions. The nonstandard system, in its turn, is reduced to an equation that contains the Hessian of the initial functional of observations. Based on this representation, a numerical algorithm for calculating the gradient of the functional considered is formulated. The procedure developed was used for the 3D model of thermodynamics. In this case, the algorithm suggested makes it possible to estimate the sensitivity of the average temperature for the selected region obtained after the assimilation with respect to the heat fluxes on the ocean surface.

\section{ACKNOWLEDGMENTS}

This work was supported by the Russian Foundation for Basic Research (project no. 12-01-00322) and the MOISE project (INRIA, France). The studies in the Sections 4-5 were supported by the Russian Scientific Fund (project no. 14-11-00609).

\section{REFERENCES}

1. G. I. Marchuk, Adjoint Equations and Analysis of Com- 1 plex Systems (Kluwer, Dordrecht, 1995).

2. J.-L. Lions, Contrölabilité exacte perturbations et stabilisation de systèmes distribués (Masson, Paris, 1988).

3. Y. K. Sasaki, "Some basic formalisms in numerical variational analysis," Mon. Weather Rev. 98 (12), 857883 (1970).

4. G. I. Marchuk and V. V. Penenko, "Application of optimization methods to the problem of mathematical simulation of atmospheric processes and environment," in Modelling and Optimization of Complex Systems: Proc. of the IFIP-TC7 Working Conf (Springer, New York, 1978), pp. 240-252.

5. F. X. Le Dimet and O. Talagrand, "Variational algorithms for analysis and assimilation of meteorological observations: Theoretical aspects," Tellus 38A, 97-110 (1986).

6. I. M. Navon, "Variational data assimilation, optimal parameter estimation and sensitivity analysis for environmental problems," in Computational Mechanics'95 (Springer, New York, 1995), Vol. 1, pp. 740-746.

7. V. I. Agoshkov and G. I. Marchuk, "On solvability and numerical solution of data assimilation problems," Russ. J. Numer. Anal. Math. Modell. 8 (1), 1-16 (1993).

8. V. I. Agoshkov, E. I. Parmuzin, and V. P. Shutyaev, "Numerical algorithm for variational assimilation of sea surface temperature data," Comput. Math. Math. Phys. 48 (8), 1293-1312 (2008).

9. E. Blayo, J. Blum, and J. Verron, "Assimilation variationnelle de données en océanographie et réduction de la dimension de l'espace de contröle," in Équations aux dérivées partielles et applications (Elsevier, Paris, 1998), pp. 205-219.

10. R. Glowinski and J. L. Lions, "Exact and approximate controllability for distributed parameter systems," Acta Numerica 1, 269-378 (1994).

11. F.-X. Le Dimet, I. M. Navon, and D. N. Daescu, "Second-order information in data assimilation," Mon. Weather Rev. 130 (3), 629-648 (2002).

12. F.-X. Le Dimet, H.-E. Ngodock, B. Luong, and J. Verron, "Sensitivity analysis in variational data assimilation,” J. Meteorol. Soc. Jpn. 75 (1B), 245-255 (1997).

13. F.-X. Le Dimet and V. Shutyaev, "On deterministic error analysis in variational data assimilation," Nonlinear Processes Geophys. 12, 481-490 (2005).

14. J.-L. Lions, Contrøle optimal de systèmes gouvernes par des équations aux dérivées partielles (Optimal Control of Systems Governed by Partial Differential Equations), (Dunod/Gauther-Villars, Paris, 1968).

15. G. I. Marchuk, V. I. Agoshkov, and V. P. Shutyaev, Adjoint Equations and the Method of Perturbations in 1 Nonlinear Problems of Mathematical Physics (Nauka, Moscow, 1993) [in Russian].

16. L. S. Pontryagin, V. G. Boltyanskii, R. V. Gamkrelidze, and E. F. Mischenko, The Mathematical Theory of Optimal Processes (Wiley, New York, 1962). 
17. F. Rabier and P. Courtier, "Four-dimensional assimilation in the presence of baroclinic instability," Q. J. R. Meteorol. Soc. 118, 649-672 (1992).

18. V. P. Shutyaev, Control Operators and Iteration Algorithms in Problems of Variational Data Assimilation (Nauka, Moscow, 2001) [in Russian].

19. W. C. Thacker, "The role of the Hessian matrix in fitting models to measurements," J. Geophys. Res. 94 (C5), 6177-6196 (1989).

20. I. Gejadze, F.-X. Le Dimet, and V. P. Shutyaev, "On analysis error covariances in variational data assimilation," SIAM J. Sci. Comput. 30 (4), 1847-1874 (2008).

21. I. Gejadze, F.-X. Le Dimet, and V. P. Shutyaev, "On optimal solution error covariances in variational data assimilation problems," J. Comput. Phys. 229, 21592178 (2010).

22. I. Yu. Gejadze, G. J. M. Copeland, F.-X. Le Dimet, and V. Shutyaev, "Computation of the analysis error covariance in variational data assimilation problems with nonlinear dynamics," J. Comput. Phys. 230, 7923-7943 (2011).

23. I. Gejadze, V. P. Shutyaev, and F.-X. Le Dimet, "Analysis error covariance versus posterior covariance in vari- ational data assimilation,” Q. J. R. Meteorol. Soc. 139, 1826-1841 (2013).

24. D. G. Cacuci, "Sensitivity theory for nonlinear systems: II. Extensions to additional classes of responses," J. Math. Phys. 22, 2803-2812 (1981).

25. A. L. Dontchev, Perturbations, Approximations and Sensitivity Analysis of Optimal Control Systems (Lecture Notes in Control and Information Sciences, No. 52) (Springer, Berlin, 1983).

26. G. I. Marchuk, V. P. Dymnikov, and V. B. Zalesny, Mathematical Models in Geophysical Hydrodynamics and Numerical Methods for Their Realization (Gidrometeoizdat, Leningrad, 1984) [in Russian].

27. V. V. Alekseev and V. B. Zalesny, "Numerical model of large-scale dynamics of the ocean," in Computational processes and systems (Nauka, Moscow, 1993), pp. 232-253 [in Russian].

28. V. I. Agoshkov, E. I. Parmuzin, and V. P. Shutyaev, "Observational data assimilation in the problem of Black Sea circulation and sensitivity analysis of its solution," Izv., Atmos. Ocean. Phys. 49 (6), 592-602 (2013).

Translated by L. Mukhortova

SPELL: 1. adjoint, 2. apriori 